\title{
(C) OPEN ACCESS \\ Nursing skill mix in European hospitals: cross-sectional study of the association with mortality, patient ratings, and quality of care
}

\author{
Linda H Aiken, ${ }^{1}$ Douglas Sloane, ${ }^{1}$ Peter Griffiths, ${ }^{2}$ Anne Marie Rafferty, ${ }^{3}$ \\ Luk Bruyneel, ${ }^{4}$ Matthew McHugh, ${ }^{1}$ Claudia B Maier, ${ }^{5}$ \\ Teresa Moreno-Casbas, ${ }^{6}$ Jane E Ball, ${ }^{2}$ Dietmar Ausserhofer, ${ }^{7}$ \\ Walter Sermeus ${ }^{4}$, For the RN4CAST Consortium
}

\begin{abstract}
- Additional material is published online only. To view please visit the journal online (http://dx.doi.org/10.1136/ bmjqs-2016-005567).
\end{abstract}

For numbered affiliations see end of article.

\section{Correspondence to}

Dr Linda H Aiken, University of Pennsylvania School of Nursing, Center for Health Outcomes and Policy Research, 418 Curie Boulevard, Philadelphia, PA 19104, USA

laiken@nursing.upenn.edu

Received 7 April 2016 Revised 4 October 2016 Accepted 8 October 2016 Published Online First

10 November 2016

\section{SLinked}

- http://dx.doi.org/10.1136/ bmjas-2016-006197

CrossMark

To cite: Aiken $\mathrm{LH}$, Sloane $\mathrm{D}$ Griffiths P, et al. BMJ Qual Saf 2017:26:559-568.

\begin{abstract}
Objectives To determine the association of hospital nursing skill mix with patient mortality, patient ratings of their care and indicators of quality of care.

Design Cross-sectional patient discharge data, hospital characteristics and nurse and patient survey data were merged and analysed using generalised estimating equations (GEE) and logistic regression models.

Setting Adult acute care hospitals in Belgium, England, Finland, Ireland, Spain and Switzerland. Participants Survey data were collected from 13077 nurses in 243 hospitals, and 18828 patients in 182 of the same hospitals in the six countries. Discharge data were obtained for 275519 surgical patients in 188 of these hospitals.
\end{abstract}

Main outcome measures Patient mortality, patient ratings of care, care quality, patient safety, adverse events and nurse burnout and job dissatisfaction.

Results Richer nurse skill mix (eg, every 10-point increase in the percentage of professional nurses among all nursing personnel) was associated with lower odds of mortality (OR=0.89), lower odds of low hospital ratings from patients $(\mathrm{OR}=0.90)$ and lower odds of reports of poor quality ( $O R=0.89)$, poor safety grades $(\mathrm{OR}=0.85)$ and other poor outcomes $(0.80<\mathrm{OR}<0.93)$, after adjusting for patient and hospital factors. Each 10 percentage point reduction in the proportion of professional nurses is associated with an $11 \%$ increase in the odds of death. In our hospital sample, there were an average of six caregivers for every 25 patients, four of whom were professional nurses.

Substituting one nurse assistant for a professional nurse for every 25 patients is associated with a $21 \%$ increase in the odds of dying.
Conclusions A bedside care workforce with a greater proportion of professional nurses is associated with better outcomes for patients and nurses. Reducing nursing skill mix by adding nursing associates and other categories of assistive nursing personnel without professional nurse qualifications may contribute to preventable deaths, erode quality and safety of hospital care and contribute to hospital nurse shortages.

\section{INTRODUCTION}

Ensuring good patient outcomes in hospitals is increasingly challenging as national economic concerns, austerity spending and health system reforms converge to create hard choices in resource allocation. ${ }^{1-3}$ At its core, hospital care is labour intensive. Health reforms have made it more so by reducing hospital length of stay and diverting discretionary admissions to outpatient settings, such that the remaining inpatients have more complex care needs. Medical advances and new technologies have been more likely to increase hospital nurse staffing requirements than to decrease them. ${ }^{4}$ The growing use of intensive care beds where professional nurse staffing is highest is but one example.

Yet the notion persists in policy and management discussions that hospitals should be able to transition to a lower complement of high skilled workers like professional nurses to reduce costs following examples in other sectors of the economy. ${ }^{5} 6$ The premise is that fewer highly skilled/higher cost professional 
lower wage assistants would result in lower costs of care without adversely affecting patient outcomes, although evidence to support such contentions is lacking. Political leaders in England have recently introduced a category of hospital caregiver called 'nursing associate', akin to the enrolled nurse position which was phased out in the 1990s. ${ }^{7}{ }^{8}$ Notably, this proposal in England for a less educated caregiver (18 months of on-the-job training is proposed) has been made in the context of concern about poor quality of hospital care, ${ }^{9}{ }^{10}$ and nursing skill mix in National Health Service (NHS) hospitals already being one of the lowest in Europe. ${ }^{11}$

Policy debates in Europe about hospital skill mix are happening in a context where evidence is limited and there is substantial variation in skill mix between and within countries. $^{3}{ }^{12-15}$ Hospital nursing skill mix varies from a high of $82 \%$ professional nurses in Germany to a low of $57 \%$ professional nurses in England and 54\% in Spain. ${ }^{16}$ Variation in hospital nurse skill mix within countries is great as illustrated in NHS hospitals in England where nursing skill mix varies from a high of $79 \%$ professional nurses in some hospitals to a low of $47 \%$ in others. ${ }^{17}$

The quality of evidence about the outcomes of nursing skill mix in Europe is very limited as noted in a Cochrane Collaboration systematic review. ${ }^{4}{ }^{18}$ Jarman et $a l^{19}$ research of English hospitals 15 years ago, remaining one of the few multihospital studies of the outcomes of nursing skill mix in Europe, found that higher proportions of auxiliary nurses (nurse assistants with limited training) were associated with higher hospital mortality. Similarly, a more recent study of NHS hospitals confirmed that more healthcare support workers (ie, nurse assistants) were associated with higher mortality. ${ }^{20}$ Most research on nursing skill mix has been of the US hospitals, where applicability to the European context is unknown. ${ }^{21-24}$ The US studies have generally concluded that a hospital skill mix with proportionately more professional nurses yields greater value because higher wages of professional nurses are offset by reductions in length of stay, lower use of intensive care, fewer costly adverse events such as hospital-acquired infections and lower readmission rates. ${ }^{25-29}$ Needleman et al estimated that holding constant the total number of hours of nursing care provided, replacing lower qualified licensed practical nurses with fewer years of education with professional nurses would result in lower hospital mortality, shorter length of stay, fewer complications and net savings. ${ }^{30}$

The aim of this paper is to inform managerial and policy decision-making about hospital nursing skill mix in Europe through an analysis of the association between nursing skill mix and patient mortality, patient ratings of their care and quality of care indicators in hospitals in six European countries: Belgium, England, Finland, Ireland, Spain and Switzerland. We mix and factors associated with professional nurse retention in hospital bedside care, important in view of evidence of present and future hospital nurse shortages in Europe. ${ }^{31}$ Our study adds substantially to the empirical evidence by its multicountry focus and the use of analytic strategies to disentangle nursing skill mix from total staffing-a limitation of previous research. Moreover, for the first time, we evaluate nursing skill mix while taking account of the educational qualifications of professional nurses, and in the context of the quality of hospital work environments that potentially enhance or undermine nurse productivity. $^{32}$

\section{DATA AND METHODS}

Data include patient discharge data by hospital, hospital administrative information and nurse and patient surveys from the six countries in the RN4CAST study that had data from all four sources-Belgium, England, Finland, Ireland, Spain and Switzerland. This is the largest data-based study of its kind. For analyses of nurse-reported outcomes, data were from 13077 nurses in a representative sample of 243 hospitals across the six countries. For analyses of patient mortality, data were from a subset of 188 of the 243 hospitals (77\%) for which detailed individual-level patient outcomes were available and included 275519 surgical patients. The analysis of patient ratings of care included a subset of 182 of the 243 hospitals (75\%) in which 18828 patients were surveyed.

Detailed accounts of the design of the 12-country RN4CAST study, the sampling of hospitals, the surveys of patients and nurses and the assembly of the surgical patient discharge records have been provided in prior reports. ${ }^{11} 3334$ The most salient features of the design and methods as they pertain to the six countries included in the present analysis are repeated here.

\section{Hospital, nurse and patient samples}

Representative samples of at least 30 general acute care hospitals with at least 100 beds in each country were selected for the nurse surveys, which were conducted in 2009-2010. The number of hospitals ranged from all 30 hospitals in Ireland to 67 in Belgium. Medical and surgical wards were randomly sampled in each hospital and all professional nurses providing direct patient care in these wards were surveyed. In Belgium, Finland and Switzerland, patients in the same hospitals and on the same wards as the nurses were also surveyed, and in Ireland and Spain patients were surveyed from subsets of the hospitals in which nurses were surveyed. In England, all adult patients discharged from study hospitals between June and August 2010 were surveyed. In five of the six countries-all except England-the patient survey instrument used was the US Agency for Healthcare 
Assessment of Healthcare Providers and Systems (HCAHPS) survey. ${ }^{35}$ Patient surveys were undertaken between 2009 and 2010, and involved a 1-day census of patients on study units in each hospital that were able to participate and who understood one of the eight questionnaire languages available. In England, we analysed data from study hospitals using the 2010 NHS Adult Inpatient Survey. ${ }^{36}$ Nurse response rates in the six European countries averaged 58\%, and the response rates for patients averaged $51 \%$.

Mortality data are for postoperative patients discharged from study hospitals in the year most proximate to the nurse survey, which ranged across countries from 2007 to $2009 .^{33}$ We included all patients discharged from the study hospitals 50 years of age and older who stayed in the hospital for at least 2 days and underwent common general, orthopaedic or vascular surgery, and for whom complete data were available on comorbidities present on admission, surgery type, discharge status and other variables used for risk adjustment. Common surgeries and comorbidities were defined following procedures published previously. ${ }^{22} 3738$ Data were coded in all countries following a standard protocol using variants of the 9th or 10 th version of the International Classification of Diseases. $^{39}$

\section{Patient involvement}

Patients were not participants in the initial design of the overall study but were active participants in the development of measures of patients' experiences with care used in the study. In developing the HCAHPS survey, AHRQ conducted patient interviews, patient focus groups, patient testing of items and numerous small-scale field tests. ${ }^{40}$ Further, Centers for Medicare and Medicaid Services ${ }^{41}$ offered three opportunities for public comment on HCAHPS and responded to over 1000 comments before finalising the instrument used in 5 of 6 countries in this study. The Picker Institute, developers of the NHS Adult Inpatient Survey used by this study in England, employed patient focus groups and cognitive interviews with patients during pilot testing. Patients were offered one page to describe what they thought of the inpatient questionnaire and which aspects of patient care were most important to them. The qualitative research did not identify major questions missing from the survey but it did lead to minor modifications that were incorporated. ${ }^{42}$

We further engaged patients in Europe to ensure that the HCAHPS survey was understandable by patients in their native languages and included aspects of patient care that were most important to them. Each country team recruited 7-12 patients who had experienced a hospital admission within the year to rate the HCAHPS questions; a total of 68 patients
HCAHPS high ratings on understandability and relevance. $^{43}$

Patients in our study are anonymous. We have a detailed plan to disseminate the study results through print, broadcast and social media in every participating country. We gratefully acknowledged the contributions of participating patients in the acknowledgement section.

\section{Primary measures}

Hospital nurse characteristics

We used four different measures of the nurse characteristics of study hospitals, each of which were derived by aggregating nurse responses to survey questions to the hospital level. Staffing measures were calculated for day shift only because of substantial variation in night staffing. Nursing skill mix was calculated for each hospital by dividing the number of professional nurses by the number of direct care nursing personnel of all qualifications that each nurse reported were present on their unit on their last shift, and averaging those ratios across all nurse respondents in each hospital. Higher ratios indicate a richer skill mix. Professional nurses in Europe meet minimum standards published by the European Commission including at least 10 years of general education at the secondary level plus 3 years of nursing education that can be in a vocational setting or university. The primary differentiation of professional nurses in Europe is whether they have bachelor's qualifications or not. Training requirements and regulations governing nurse assistants vary widely across hospitals in Europe and our measure does not differentiate nurse assistants by the specifics of their qualifications. ${ }^{44-45}$ Total staffing was calculated by dividing the total number of nursing personnel (including professional nurses and others) by the number of patients that each nurse reported were present on their unit on their last shift, and averaging those ratios across all nurse respondents in each hospital. These averages were then multiplied by 25 , the average number of patients per unit, so the resulting numbers indicate how many staff were present for every 25 patients across each hospital. Higher ratios indicate more favourable staffing. Nurse education was the percentage of all professional nurses in each hospital reporting they had a bachelor's degree. The nurse work environment in each hospital was measured using the Practice Environment Scale of the Nursing Work Index (PES-NWI), an internationally validated measure. ${ }^{46} 47$ The PES-NWI measures modifiable organisational behaviours that comprise five subscales indicating: (1) managerial support for nursing, (2) nurse participation in hospital affairs, (3) doctor-nurse relations, (4) promotion of care quality and (5) staffing and resource adequacy. We measure the hospital work environment by averaging across all nurses in each 
items comprising the first four subscales. The staffing and resource adequacy subscale was dropped because of its high correlation with the direct measures of nurse staffing included in the analytic models as in previous studies. $^{32}$

Our estimates of nursing skill mix and total staffing in the different hospitals involve estimates from nurses working different shifts on different units on different days. Thus, it is not possible with our data to determine the consistency between nurses on the same shift on the same unit in the same hospital on the same day. But with reasonably large numbers and in many cases very large numbers of nurses in all of our study hospitals, we are confident that when we will aggregate these numbers across nurses to the hospital level we will get reasonable estimates of skill mix and total staffing.

Other hospital characteristics

In analyses of both patient outcomes and nurse-reported outcomes we controlled for hospital size, technology and teaching status. Hospital size was the number of beds. Teaching status distinguished hospitals that were non-teaching (no medical residents or fellows) and teaching hospitals (which had residents or fellows). High technology hospitals had facilities for open-heart surgery, major organ transplants or both. Dummy variables account for unmeasured differences across countries.

Patient mortality

Patient discharge records allow us to determine whether patients died in the hospital within 30 days of admission. In our analyses of the associations between hospital nursing characteristics and the likelihood of dying, we risk adjust those likelihoods by controlling for patient age, sex, admission type (emergency/elective), 43 dummy variables indicating surgery type and 17 dummy variables indicating comorbidities present on admission, which are included in the Charlson Comorbidity Index. ${ }^{33} 48$

\section{Patient ratings}

In every country except England, patients were asked to rate their hospitals on a scale from 0 (worst possible) to 10 (best possible). In England, patients were asked whether they would rate the care they received in their hospital as excellent, very good, good, fair or poor. We classified patients as giving low ratings to their hospital if, in England, they described their care as anything less than excellent, or if, in the other countries, they scored their hospital as 8 or less. This corresponds to the manner in which patient ratings are 'top-coded' in the HCAHPS survey. ${ }^{35}$

\section{Nurse-reported outcomes}

Nine nurse-reported outcomes were measured by creating simple dichotomous variables. Low or poor quality of patient care was assessed as the proportion the quality of care on their units was fair or poor. Nurse-assessed quality of care has been shown to be highly related to independent measures of patient outcomes such as mortality. ${ }^{49}$ Quality of care was also indirectly assessed as the proportion of nurses who would recommend their hospital to friends and family. Low or poor patient safety grade was assessed as the proportion of nurses who gave their ward a poor or fair safety grade. Patient safety culture was assessed using responses to seven items which, like patient safety grade, was derived from the Agency for Healthcare Research and Quality Hospital Survey on Patient Safety Culture, and which asked nurses whether they felt their mistakes were held against them, whether important patient information is lost during shift changes and whether the actions of hospital management show that patient safety is a top priority, among others. ${ }^{50}$ Poor patient safety culture was assessed as the proportion of nurses whose responses to three or more of the seven items indicated unsafe conditions. We assessed nurse-reported adverse events by calculating the proportions of nurses who reported that (1) pressure ulcers, (2) falls with injuries and (3) urinary tract infections occurred occasionally or frequently (combining 'a few times a month', 'once a week', 'a few times a week' and 'every day') rather than rarely or never (combining categories 'never', 'a few times a year or less' and 'once a month or less'). Nurse job dissatisfaction was assessed using a single item which asked nurses how satisfied they were with their current job. Nurse burnout was measured with the emotional exhaustion subscale of the Maslach Burnout Inventory, ${ }^{51}$ an instrument with established reliability and validity in international research. ${ }^{52}$ We assessed these factors as the proportion of dissatisfied nurses, and the proportion of nurses with high burnout (scores above 27 on the emotional exhaustion subscale).

\section{Analytic methods}

Descriptive information on the variables of interest is provided, and shows how skill mix, total bedside caregiver staffing, nurse practice environments and nurse education vary across different hospitals. The association of nursing skill mix is then estimated for patient mortality, patient ratings of their hospitals and nurse reports of quality of care, patient safety, adverse events and nurse burnout and dissatisfaction, before and after controlling for other hospital characteristics, patient characteristics (in the mortality analyses) and nurse characteristics (in the analyses of nurse-reported outcomes). Other hospital characteristics controlled included total nursing personnel staffing, nurse education and the nurse practice environment, as well as size, teaching status and technology. Patient characteristics included age, sex, admission type, surgery type and comorbidities present on admission. Nurse 
status and unit specialty. Dummy variables representing the different countries were also included in the models with controls to allow for unmeasured differences across countries. Generalised estimating equations (GEE) were used in analysing mortality and logistic regression models were used in analysing patient ratings and nurse-reported outcomes. In both approaches, we took account of patients being nested within hospitals. We tested for interactions but found none. We also dropped from the models the association of the nurse practice environment with mortality, since it was insignificant, and the association of nurse education with patient ratings and nurse-reported outcomes, for the same reason. In online supplementary tables in the appendix, we show the correlations between nursing characteristics and other hospital characteristics, and the relationships of the full set of nursing and hospital characteristics with the different outcomes.

\section{RESULTS}

As noted in table 1, there were 275519 surgical discharges from the 188 study hospitals for which patient data were available, an average of 1466 discharges per hospital. There were 3569 surgical patient deaths-an average of 19 deaths per hospital during the study period-yielding an average hospital mortality rate of 12.8 per 1000 discharges. The middle panel of table 1 shows that 18828 patients were surveyed in 182 hospitals, for an average of just over 100 patient respondents per hospital. In the average hospital, 54\% of the patients surveyed gave their hospitals low ratings. The bottom panel shows that 13077 professional nurses were surveyed in the 243 study hospitals. The average hospital had 54 nurse respondents. On average, slightly more than one in five nurses rated the quality of care on their hospital unit as poor or fair, and while only an average of $7 \%$ of the nurses across hospitals gave their hospital a poor or failing safety grade, one-third indicated that their hospital exhibited a poor safety culture. Nearly one in five nurses would not recommend their hospital to friend or family, and on average $9 \%$ of the nurses reported that pressure ulcers occurred in their hospitals occasionally or frequently, and $12 \%$ and $23 \%$ said the same about falls with injury and urinary tract infections, respectively. In the average hospital, almost $30 \%$ of nurses scored high on the burnout scale, and a similar per cent expressed dissatisfaction with their job.

Table 2 shows that overall the skill mix, or the percentage of professional nurses among the total nursing personnel in this sample of hospitals, was roughly 66\%, and ranged across hospitals from 41\% to $87 \%$. Total nursing personnel staffing overall was roughly 6.1 nursing personnel (all qualifications) for every 25 patients, and ranged from 2.7 per 25 patients in the poorest staffed hospital to 13.8 per 25 patients in the best. Practice environment scores averaged roughly 2.7 across the 188 hospitals-slightly above the middle of the 4-point scale-and ranged from 2.1 in the hospital with the worst environment to 3.4 in the hospital with the best. Nurse education, or the percentage of bachelor's prepared nurses among all professional nurses, averaged $47 \%$ overall, and ranged widely from $0 \%$ in some hospitals to $100 \%$ in others.

Table 3 shows the associations of skill mix with inpatient mortality, patient ratings of their hospitals

Table 1 Patients discharged, patients surveyed and nurses surveyed in the study hospitals in six European countries, and descriptive information on outcomes derived from them (RN4CAST data)

\begin{tabular}{|c|c|c|c|c|c|}
\hline & Total & Hospital mean & SD & 25th percentile & 75th percentile \\
\hline Patient discharges & 275519 & 1466 & 1093 & 713 & 1947 \\
\hline Patient deaths & 3569 & 19.0 & 19.8 & 6.5 & 25.0 \\
\hline Patient mortality rate (deaths per 1000 discharges) & & 12.8 & 9.1 & 8.0 & 16.2 \\
\hline Patient survey respondents & 18828 & 103 & 139 & 29 & 77 \\
\hline Percent giving hospital low ratings & & $54 \%$ & $15 \%$ & $39 \%$ & $60 \%$ \\
\hline Nurse survey respondents & 13077 & 54 & 27 & 37 & 65 \\
\hline \multicolumn{6}{|l|}{ Percent of nurses reporting } \\
\hline Poor/fair unit quality & & $22 \%$ & $14 \%$ & $11 \%$ & $32 \%$ \\
\hline Poor/failing safety grade & & $7 \%$ & $6 \%$ & $2 \%$ & $9 \%$ \\
\hline Poor safety culture & & $34 \%$ & $16 \%$ & $24 \%$ & $45 \%$ \\
\hline Would not recommend hospital to friends or family & & $18 \%$ & $12 \%$ & $8 \%$ & $24 \%$ \\
\hline Pressure ulcers & & $9 \%$ & $8 \%$ & $3 \%$ & $14 \%$ \\
\hline Falls with injury & & $12 \%$ & $9 \%$ & $5 \%$ & $15 \%$ \\
\hline Urinary tract infections & & $23 \%$ & $12 \%$ & $15 \%$ & $29 \%$ \\
\hline High burnout & & $30 \%$ & $17 \%$ & $15 \%$ & $42 \%$ \\
\hline Job dissatisfaction & & $31 \%$ & $16 \%$ & $17 \%$ & $42 \%$ \\
\hline
\end{tabular}

Patient discharge data (used for the mortality analyses) were available for 188 (77\%) of the 243 hospitals in which nurses were surveyed. Patient survey 
Table 2 Nursing characteristics in the study hospitals in six European countries

\begin{tabular}{lcccc}
\hline Nursing characteristics & Mean & SD & 25th percentile & 75th percentile \\
\hline Skill mix (\% professional nurses) & $65.6 \%$ & $9.8 \%$ & $56.4 \%$ & $74.1 \%$ \\
Total staffing & 6.09 & 1.61 & 5.01 & 7.10 \\
Practice environment & 2.67 & 0.24 & 2.51 & 2.82 \\
Nurse education (\% bachelors) & $46.8 \%$ & $26.3 \%$ & $26.9 \%$ & $68.0 \%$ \\
\hline
\end{tabular}

Skill mix is the percentage of professional nurses among all nursing personnel in the hospital. Total staffing is the total number of all nursing personnel (at all qualification levels) for every 25 patients they cared for. The practice environment measure is the average score for each hospital across four subscales indicating (1) managerial support for nursing, (2) nurse participation in hospital affairs, (3) doctor-nurse relations and (4) promotion of care quality (where a score of 1 would indicate extremely poor on all subscales, and a score of 4 would indicate excellent on all subscales). Nurse education is the percentage of all professional nurses in each hospital with bachelor's degrees.

Table 3 ORs indicating the association of nursing skill mix with inpatient mortality, patient ratings of their hospitals, nurse-reported quality of care and nurse outcomes in hospitals in six European countries

\begin{tabular}{|c|c|c|c|c|}
\hline \multirow[b]{3}{*}{ Outcome } & \multicolumn{4}{|c|}{ ORs reflecting the associations of skill mix with the different outcomes } \\
\hline & \multicolumn{2}{|l|}{ Without controls } & \multicolumn{2}{|l|}{ With controls } \\
\hline & OR $95 \% \mathrm{Cl}$ & p Value & OR $95 \% \mathrm{Cl}$ & p Value \\
\hline 30-day inpatient mortality & $0.90(0.81$ to 1.00$)$ & 0.058 & $0.89(0.80$ to 0.98$)$ & 0.018 \\
\hline Low hospital rating by patients & $0.81(0.74$ to 0.88$)$ & $<0.001$ & $0.90(0.81$ to 0.99$)$ & 0.026 \\
\hline Poor/fair unit quality & $0.90(0.81$ to 1.00$)$ & 0.053 & $0.89(0.80$ to 0.98$)$ & 0.016 \\
\hline Poor/failing safety grade & $1.05(0.93$ to 1.17$)$ & 0.457 & $0.85(0.73$ to 0.99$)$ & 0.040 \\
\hline Poor safety culture & $1.09(1.01$ to 1.15$)$ & 0.022 & $0.93(0.87$ to 0.99$)$ & 0.027 \\
\hline Nurse would not recommend hospital & $0.97(0.87$ to 1.08$)$ & 0.616 & $0.82(0.72$ to 0.93$)$ & 0.001 \\
\hline Pressure ulcers & $0.82(0.74$ to 0.91$)$ & 0.001 & $0.85(0.73$ to 0.98$)$ & 0.027 \\
\hline Falls with injury & $0.89(0.79$ to 1.00$)$ & 0.058 & $0.80(0.71$ to 0.91$)$ & 0.001 \\
\hline Urinary tract infections & $0.89(0.83$ to 0.96$)$ & 0.002 & $0.88(0.78$ to 1.00$)$ & 0.049 \\
\hline High nurse burnout & $0.93(0.85$ to 1.01$)$ & 0.102 & $0.89(0.80$ to 1.00$)$ & 0.043 \\
\hline Nurse job dissatisfaction & $0.87(0.79$ to 0.95$)$ & 0.003 & $0.91(0.83$ to 0.99$)$ & 0.025 \\
\hline
\end{tabular}

Inpatient mortality was measured using patient discharge data, hospital rating by patients was measured using patient survey data and the remainder were measured using nurse survey data. Huber-White Cluster corrections were used to estimate SEs associated with the skill mix coefficients. Skill mix was measured in units of 10 percentage points. Controls in the mortality models include hospital characteristics (total bedside care staffing, nurse education, teaching status, high technology and bed size), patient characteristics (age, sex, admission type, 43 dummy variables indicating surgery type and 17 dummy variables indicating comorbidities present on admission) and country. Controls in the models estimating patient ratings included the same hospital characteristics and country. Controls in the models estimating nurse-reported outcomes included hospital characteristics (total nursing personnel staffing, nurse practice environment, teaching status, high technology and bed size) nurse characteristics (age, sex, full-time employment status and unit specialty) and country.

and the nine different nurse-reported outcomes before and after adjusting for differences across hospitals in patient and nurse characteristics and for other hospital characteristics of importance (total staffing, nurse education, the nurse work environment, size, teaching status and technology) and unmeasured differences across countries. Before adjustment (or without controls), hospitals with richer skill mixes appear to have higher odds on a couple of these 11 outcomes (involving poor safety grades and safety cultures) but lower odds on the others, and for 6 of the 11 outcomes the ORs are insignificant $(p>0.05)$. While controlling for the various potentially confounding characteristics affects the skill mix association with these 11 outcomes differently-some increase, others decrease, and some hardly change at all-after adjustment hospitals with richer nursing skill mixes have lower odds on all 11 of the unfavourable outcomes and the differences are significant in every case. Every 10-point increase in the percentage of professional nurses among all nursing personnel diminishes the odds on patients dying by a factor of 0.89 and diminishes the odds on patients giving their hospitals low ratings by a factor of 0.90 . And nursing skill mix is similarly related to all nine of the different nurse-reported outcomes. Net of the other nursing characteristics, and after taking account of unmeasured differences between countries and measured differences in nurse characteristics and other hospital characteristics that might affect nurse reports, nurses in hospitals with richer skill mixes have lower odds on reporting poorer quality care, lower patient safety, high burnout and job dissatisfaction. They also are more likely to recommend their hospital, less likely to report inadequate safety culture in their hospital and less likely to report occasional or 
frequent pressure ulcers, falls with injuries and urinary infections involving their patients. The ORs associated with the effect of nursing skill mix on these different outcomes, which range from 0.80 for reporting falls with injury to 0.93 for reporting that their hospital has a poor safety culture, imply that such reports differ by somewhere between $7 \%$ and $20 \%$ in hospitals that differ by $10 \%$ in the proportion of professional nurses among all nursing personnel in the hospital.

\section{DISCUSSION}

In a context in Europe in which hospital nurse staffing is a frequent target for budget reductions, and policy makers are suggesting the introduction of lower skilled and less educated nurse substitutes like 'nursing associates', our results suggest caution is warranted. We find a nursing skill mix in hospitals with a higher proportion of professional nurses is associated with significantly lower mortality, higher patient ratings of their care and fewer adverse care outcomes. Specifically, each $10 \%$ increase in the proportion of nursing personnel who are professional nurses is associated with an $11 \%$ decrease in the odds of patient deaths after general surgery. Or from the perspective of reducing skill mix, which is more in vogue among policy makers, each $10 \%$ reduction in the proportion of professional nurses is associated with a $12 \%$ increase in the odds of patient deaths. We note that the proportion of professional nurses can be altered by either reducing the number of professional nurses or adding less skilled workers, both of which have the same effect on reducing skill mix and risking poor patient outcomes. Additional research is needed to establish that an increase in the proportion of professional nurses will have the aforementioned effect, since these estimates are inferred from cross-sectional data which make causal inferences less certain.

Our research suggests that substituting lower qualified personnel for professional nurses may result in an increase in preventable deaths and other adverse outcomes for patients. In our representative sample of European hospitals, there was an average of six nursing personnel for every 25 patients, four of whom were professional nurses. The effect of substituting one nurse assistant for one professional nurse to care for every 25 patients-thus reducing the skill mix from $66.7 \%$ to $50 \%$, or by $16.7 \%$-would be to increase the odds on mortality by $21 \%$ (or, mathematically, by a factor of $\left.(1 / 0.89)^{1.667}=1.21\right)$. Alternatively, the effect of substituting one professional nurse for one nurse assistant to care for every 25 patients-thus increasing the skill mix from $66.7 \%$ to $83.3 \%$, or by the same $16.7 \%$ - would be to decrease the odds on mortality by $18 \%$ (or by a factor of $0.89^{1.667}=0.82$ ). Other adverse outcomes would be similarly affected. For example, every $10 \%$ increase in the percentage of associated with a 10\% reduction in the odds of patients giving their hospital poor ratings. Total staffing, or the total number of nursing personnel per patient, was not a significant predictor of how patients rated their hospitals, only the proportion of professional nurses.

Our results also showed that nurses in hospitals with a greater proportion of professional nurses (higher skill mix) were less likely to rate the quality of care in their hospital as fair or poor, were less likely to report poor patient safety culture and less likely to have misgivings about recommending the hospital to family members and friends. Additionally nurses in hospitals with a rich nursing skill mix were less likely to report common adverse patient events such as falls with injuries, pressure ulcers and urinary tract infections. Previous research has established that nurse reports of quality are significantly associated with actual patient outcomes. ${ }^{49}$

Paradoxically, despite policy and managerial interest in professional nurse substitutes driven by budget constraints, there are also concerns about existing and future shortages of professional nurses in Europe. ${ }^{53}$ Our results suggest that in hospitals with richer skill mix, professional nurses are less likely to experience high job-related burnout-a patient safety hazard and nurse retention problem-or to be dissatisfied with their jobs potentially contributing to expensive and disruptive turnover and nurse shortage at the hospital bedside.

While this study is the largest and most comprehensive study of the outcomes of hospital nurse skill mix in Europe to date, some limitations should be noted. Given our study design, we are able to link nurses and patients to the same hospitals, but we cannot link specific nurses and patients, so results must be interpreted with some caution. The study is cross-sectional offering a snapshot of associations between hospital staffing and patient outcomes at a single point in time. Thus, and as noted above, we cannot be sure of causal links between skill mix and outcomes. We did, however, test and eliminate a number of alternative explanations for the relationships found between nursing skill mix and outcomes including variation in the quality of the work environment, hospital size and technology availability. A repeat study of the same hospitals over time, following the same research protocol, could provide greater certainty that improved skill mix would result in improved patient outcomes. A common research protocol and survey instruments were used across countries except in the measurement of patient ratings of their hospitals where data from a national survey in England was used instead of the primary patient survey instrument used in other countries. To reduce the effects of the methods resulting from the different instruments, we used a comparable measure of global patient ratings of 
hospital physician staffing because uniform data across hospitals in different countries do not exist. However, in other research physician factors have been associated with patient outcomes independently of nurse staffing, and thus would not likely explain the relationships between nursing skill mix and patient outcomes. ${ }^{20} 38$ The absence of standardised qualifications for nurse assistants in Europe did not allow us to take into account the effects of differences in nurse assistants between countries, although we take account of unmeasured country differences through the use of 'country' dummy variables in our predictive models. Data availability in Europe did not permit a study of the comparative costs of nurse staff composition. The better patient outcomes associated in this study with richer skill mix suggest that, as in the USA where detailed cost information is available, fewer expensive complications like infection, shorter length of stay and fewer intensive care unit days among patients in hospitals with better professional nurse staffing would likely offset higher labour costs associated with richer skill mix and return good value to health systems. ${ }^{26}$

Our results of one of the first comprehensive studies of nursing skill mix in Europe are similar to results obtained in studies of nursing skill mix in the USA. Our four state study in the USA showed 30-day general surgery mortality rates of $1.2 \%{ }^{32}$ compared with European in-hospital mortality rates shown here of 1.28\%; the US patients studied included all adult patients aged 20-85 years while the European patients were aged 50 years and older. The US hospitals have a higher nursing skill mix than Europe averaging 75\% professional nurses, with hospitals ranging from a low of $68 \%$ to a high of $83 \%$ professional nurses. Hospital nursing skill mix in the European countries studied here averaged $66 \%$ professional nurses, varying from $41 \%$ to $87 \%$. Nevertheless, studies in the USA have reached similar conclusions to what we found in Europe, that the higher the proportion of professional nurses the better the outcomes for patients.

\section{CONCLUSIONS}

Findings from this large and unique study of nursing skill mix in European hospitals suggests that caution should be taken in implementing policies to reduce hospital nursing skill mix because the consequences can be life threatening for patients. Beyond risking preventable death among hospitalised patients, the erosion of nursing skill mix could negatively impact overall quality and safety of care as well as patients' perceptions of the adequacy of their hospitals at a time when there are growing concerns about eroding hospital care quality. ${ }^{10}$ Indeed, in this study we found that half of the almost 19000 patients surveyed failed to rate their hospitals as excellent. For hospital managers and policymakers looking for evidence of how to get the most value for investments in hospital the proportion of professional nurses as well as reducing disparities in nursing skill mix between hospitals within countries. Our study adds new and important evidence that diluting hospital nurse skill mix by adding lower skilled nurse assistants and/or reducing professional nurses is not in the public interest.

\section{Author affiliations}

${ }^{1}$ University of Pennsylvania School of Nursing, Center for Health Outcomes and Policy Research, Philadelphia,

Pennsylvania, USA

${ }^{2}$ Faculty of Health Sciences, University of Southampton, Southampton, UK

${ }^{3}$ King's College London, Florence Nightingale School of

Nursing and Midwifery, London, UK

${ }^{4}$ University of Leuven, Leuven Institute for Healthcare Policy,

Leuven, Belgium

${ }^{5}$ Department of Healthcare Management, Technische

Universitat Berlin, Berlin, Germany

${ }^{6}$ Investén-Isciii. Instituto de Salud Carlos III, Ministerio de Ciencia e Innovación, Madrid, Spain

${ }^{7}$ Universitat Basel Department Public Health, Institute of Nursing Science, Basel, BS, Switzerland

Twitter Follow Linda Aiken at @LindaAiken_Penn, Luk Bruyneel at@Luk Bruyneel, Jane Ball at@JaneEBall and Peter Griffiths at@workforcesoton

Acknowledgements We thank Tim Cheney for analytic assistance, the patients and nurses who participated in our study, and the RN4CAST research teams in the participating countries.

Contributors All of the named authors participated in data collection, the writing of the manuscript and revisions.

Funding European Union's Seventh Framework Program (223468), National Institute of Nursing Research, National Institutes of Health (NR014855), Spanish Ministry of Science and Technology.

Competing interests None declared.

Ethics approval The study was approved by the University of Pennsylvania human subjects committee.

Provenance and peer review Not commissioned; externally peer reviewed.

RN4CAST consortium Walter Sermeus (Director), Koen Van den Heede, Luk Bruyneel, Emmanuel Lesaffre, Luwis Diya (Belgium, Catholic University Leuven); Linda Aiken

(Codirector), Herbert Smith, Douglas Sloane (USA, University of Pennsylvania); Anne Marie Rafferty, Jane Ball, Simon Jones (UK, King's College London); Peter Griffiths (UK, University of Southampton); Juha Kinnunen, Anneli Ensio, Virpi Jylhä

(Finland, University of Eastern Finland); Reinhard Busse, Britta Zander, Miriam Blümel (Germany, Berlin University of Technology); John Mantas, Dimitrios Zikos, Marianna Diomidous (Greece, University of Athens); Anne Scott, Anne Matthews, Anthony Staines (Ireland, Dublin City University); Ingeborg Strømseng Sjetne (Norwegian Knowledge Centre for the Health Services) Inger Margrethe Holter (Norwegian Nurses Organization); Tomasz Brzostek, Maria Kózka, Piotr Brzyski (Poland, Jagiellonian University Collegium Medicum); Teresa Moreno-Casbas, Carmen Fuentelsaz-Gallego, Esther Gonzalez-María, Teresa Gomez-Garcia (Spain, Institute of Health Carlos III); Carol Tishelman, Rikard Lindqvist, Lisa Smeds (Sweden, Karolinska Institute); Sabina De Geest, Maria Schubert, René Schwendimann (Switzerland, Basel University); Maud Heinen, Lisette Schoonhoven, Theo van Achterberg (Netherlands, Radboud University Nijmegen Medical Centre).

Open Access This is an Open Access article distributed in accordance with the Creative Commons Attribution Non Commercial (CC BY-NC 4.0) license, which permits others to distribute, remix, adapt, build upon this work non- 
terms, provided the original work is properly cited and the use is non-commercial. See: http://creativecommons.org/licenses/by$\mathrm{nc} / 4.0 /$

\section{REFERENCES}

1 Clemens T, Michelsen K, Brand H. Supporting health systems in Europe: added value of EU actions? Health Econ Policy Law 2014;9:49-69.

2 Karanikolos M, Mladovsky P, Cylus J, et al. Financial crisis, austerity, and health in Europe. Lancet 2013;381:1323-31.

3 Antonanzas F. The impact of the economic downturn on healthcare in Spain: consequences and alternatives. Expert Rev Pharmacoecon Outcomes Res 2013;13:433-9.

4 Buchan J, O’May F, Dussault G. Nursing workforce policy and the economic crisis: a global overview. J Nurs Scholarsh 2013;45:298-307.

5 Jacob ER, McKenna L, D'Amore A. The changing skill mix in nursing: considerations for and against different levels of nurse. J Nurs Manag 2015;23:421-6.

6 Norrish BR, Rundall TG. Hospital restructuring and the work of registered nurses. Milbank Q 2001;79:55-79.

7 Seccombe I, Smith G, Buchan J, et al. Enrolled nurses: a study for the UKCC. Institute for Employment Studies, Report no. 344, 1997.

8 Gummer P. Nursing associate role offers new route into nursing. UK: Department of Health. http://www.gov.uk/government/ news/nursing-associate-role-offers-new-route-into-nursing (accessed 25 Jan 2016). NHS bursary reform and nurse education: GOV.UK, 2015.

9 Francis R. Report of the mid Staffordshire NHS foundation trust public inquiry. http://webarchive.nationalarchives.gov.uk/ 20150407084003/; http://www.midstaffspublicinquiry.com/ report (accessed 25 Jan 2016), 2013.

10 Keogh B. Review into the quality of care and treatment provided by 14 hospital trusts in England: overview report. NHS Report. http://www.nhs.uk/nhsengland/bruce-keogh-review/documents/ outcomes/keogh-review-final-report.pdf (accessed 25 Jan 2016), 2013.

11 Aiken LH, Sermeus W, Vanden Heede K, et al. Patient safety, satisfaction, and quality of hospital care: cross-sectional surveys of nurses and patients in 12 countries in Europe and the United States. BMJ 2012;344:e1717.

12 Department of Health Ireland. Taskforce on staffing and skill mix for nursing. http://health.gov.ie/future-health/office-ofchief-nursing-officer/taskforce-on-staffing-and-skillmix-for-nursing/ (accessed 15 Oct 2014). 2014.

13 Commission on Nursing. Report of The Commission on Nursing. A blueprint for the future. Dublin, 1998.

14 Robinson S, Griffiths P, Maben J. Calculating skill mix: implications for patient outcomes and costs. Nurs Manag (Harrow) 2009;16:22-3.

15 National Institute For Health And Care Excellence. Safe staffing for nursing in adult inpatient wards in acute hospitals. NICE, 2014: http://www.nice.org.uk/guidance/sg1 (accessed 19 Aug 2015).

16 Aiken LH, Sloane DM, Bruyneel L, et al. Nurses' reports of working conditions and hospital quality of care in 12 countries in Europe. Int J Nurs Stud 2013;50:143-53.

17 Ball JE, Murrells T, Rafferty AM, et al. 'Care left undone' during nursing shifts: associations with workload and perceived quality of care. BMJ Qual Saf 2014;23:116-25.

18 Butler M, Collins R, Drennan J, et al. Hospital nurse staffing
Database Syst Rev 2011. (cited 16 Jan 2016) http://www. cochrane.org/CD007019/EPOC_hospital-nurse-staffingmodels-and-patient-and-staff-related-outcomes

19 Jarman B, Simon G, Alves B, et al. Explaining differences in English hospital death rates using routinely collected data. BMJ 1999;318:1515-20.

20 Griffiths P, Ball J, Murrells T, et al. Registered nurse, health care support worker, medical staffing levels and mortality in English hospital trusts: a cross-sectional study. BMJ Open 2016;6:e008751.

21 Needleman J, Buerhaus P, Mattke S, et al. Nurse-staffing levels and the quality of care in hospitals. N Engl J Med 2002;346:1715-22.

22 Cho SH, Ketefian S, Barkauskas VH, et al. The effects of nurse staffing on adverse events, morbidity, mortality, and medical costs. Nursing Res 2003;52:71-9.

23 Blegen MA, Goode, CJ, Spetz J, et al. Nurse staffing effects on patient outcomes: safety-net and non-safety-net hospitals. Med Care 2011;49:406-14.

24 Unruh L. Licensed nurse staffing and adverse events in hospitals. Med Care 2003;41:142-52.

25 Dall TM, Chen YJ, Seifert RF, et al. The economic value of professional nursing. Med Care 2009;47:97-104.

26 Li Y-F, Wong ES, Sales AE, et al. Nurse staffing and patient care costs in acute inpatient nursing units. Med Care 2011;49:708-15.

27 Martsolf GR, Auerbach D, Benevent R, et al. Examining the value of inpatient nurse staffing: an assessment of quality and patient care costs. Med Care 2014;52:982-8.

28 McHugh MD, Berez J, Small DS. Hospitals with higher nurse staffing had lower odds of readmissions penalties than hospitals with lower staffing. Health Aff (Millwood) 2013;32:1740-7.

29 Silber JH, Rosenbaum PR, McHugh MD, et al. Comparison of the value of nursing work environments in hospitals across different levels of patient risk. JAMA Surg 2016;151:527-36.

30 Needleman J, Buerhaus PI, Stewart M, et al. Nurse staffing in hospitals: is there a business case for quality? Health Aff (Millwood) 2006;25:204-11.

31 World Health Organization Regional Office for Europe. Nursing and Midwifery, Data and Statistics. http://www.euro. who.int/en/health-topics/Health-systems/nursing-andmidwifery/data-and-statistics (accessed 25 Jan 2016), 2016.

32 Aiken LH, Cimiotti JP, Sloane DM, et al. Effects of nurse staffing and nurse education on patient deaths in hospitals with different nurse work environments. Med Care 2011;49:1047-53.

33 Aiken LH, Sloane DM, Bruyneel L, et al. Nurse staffing and education and hospital mortality in nine European countries: a retrospective observational study. Lancet 2014;383:1824-30.

34 Sermeus W, Aiken LH, Van den Heede K, et al. Nurse Forecasting in Europe (RN4CAST): rationale, design and methodology. BMC Nurs 2011;10:6.

35 Giordano LA, Elliott MN, Goldstein E, et al. Development, implementation, and public reporting of the HCAHPS survey. Med Care Res Rev 2010;67:27-37.

36 National NHS Patient Survey Programme. Survey of adult inpatients 2010. NHS Surveys: UK http://www.nhssurveys.org/ survey/1017 (accessed 31 Jan 2016], 2011.

37 Silber JH, Kennedy SK, Even-Shoshan O, et al. Anesthesiologist direction and patient outcomes. Anesthesiology 2000;93:152-63.

38 Aiken LH, Clarke SP, Sloane DM, et al. Hospital nurse staffing and patient mortality, nurse burnout, and job dissatisfaction. JAMA 2002;288:1987-93.

39 Quan H, Sundararajan V, Halfon P, et al. Coding algorithms for defining comorbidities in ICD-9-CM and ICD-10 administrative data. Med Care 2005;43:1130-9. 
40 Sofaer S, Crofton C, Goldstein E, et al. What do consumers want to know about the quality of care in hospitals? Health Serv Res 2005;40(Pt 2):2018-36.

41 Centers for Medicare and Medicaid Services. HCAHPS: Patients' perspectives of care survey. https://www.cms.gov/ Medicare/Quality-Initiatives-Patient-Assessment-instruments/ HospitalQualityInits/HospitalHCAHPS.html (accessed 29 Jan 2016), 2014.

42 Reeves R, Coulter A, Jenkinson C, et al. Development and pilot testing of questionnaires for use in the acute NHS trust inpatient survey programme. Europe: Picker Institute, http://www. nhssurveys.org/Filestore/documents/DevelopmentInpatient Questionnaire.pdf (accessed 29 Jan 2016), 2002.

43 Squires A, Bruyneel L, Aiken LH, et al. Cross-cultural evaluation of the relevance of the HCAHPS survey in five European countries. Int J Qual Health Care 2012;24:470-5.

44 Hewko SJ, Cooper SL, Huynh H, et al. Invisible no more: a scoping review of the health care aide workforce literature. BMC Nurs 2015;14:38.

45 Netherlands Institute for Health Services Research. Consortium for the study on the support for the definition of core competences of healthcare assistants (CC4HCA). Report on the preliminary results. Leuven: KU Leuven.http://www. nivel.nl/en/cc4hca (accessed 10 Jul 2016), 2014.
46 Lake ET. The nursing practice environment: measurement and evidence. Med Care Res Rev 2007;64(2 Suppl): 104S-22S.

47 Warshawsky NE, Havens DS. Global use of the practice environment scale of the nursing work index. Nurs Res 2011;60:17-31.

48 Charlson ME, Pompei P, Ales KL, et al. A new method of classifying prognostic comorbidity in longitudinal studies: development and validation. J Chronic Dis 1987;40:373-83.

49 McHugh MD, Stimpfel AW. Nurse reported quality of care: a measure of hospital quality. Res Nurs Health 2012;35:566-75.

50 Sorra J, Nieva V. Hospital survey on patient safety culture. Rockville: Agency for Healthcare Research and Quality, 2004.

51 Maslach C, Jackson SE. Maslach burnout inventory manual. 2nd edn. Palo Alto, CA: Consulting Psychologists Press, 1986.

52 Poghosyan L, Aiken LH, Sloane DM. Factor structure of the Maslach burnout inventory: an analysis of data from large scale cross-sectional surveys of nurses from eight countries. Int J Nurs Stud 2009;46:894-902.

53 BBC News. More than 10,000 nursing posts unfilled in London. London: BBC, 2016. http://www.bbc.com/news/ uk-england-london-35242993 (accessed 25 Jan 2016). 